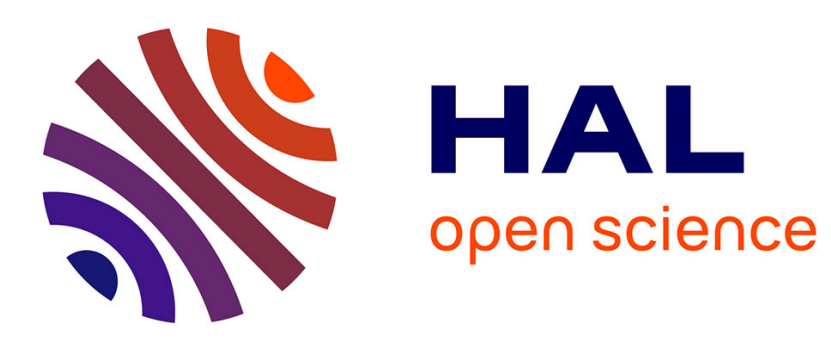

\title{
La double vue. Sur le voyage en Égypte (1869) de Théophile Gautier
}

Sarga Moussa

\section{To cite this version:}

Sarga Moussa. La double vue. Sur le voyage en Égypte (1869) de Théophile Gautier. Le Temps des médias. Revue d'histoire, 2007, 8, pp.34-45. hal-00910107

\section{HAL Id: hal-00910107 \\ https://hal.science/hal-00910107}

Submitted on 1 Dec 2013

HAL is a multi-disciplinary open access archive for the deposit and dissemination of scientific research documents, whether they are published or not. The documents may come from teaching and research institutions in France or abroad, or from public or private research centers.
L'archive ouverte pluridisciplinaire HAL, est destinée au dépôt et à la diffusion de documents scientifiques de niveau recherche, publiés ou non, émanant des établissements d'enseignement et de recherche français ou étrangers, des laboratoires publics ou privés. 


\section{La double vue. Sur le voyage en Égypte (1869) de Théophile Gautier}

On connaît l'importance de la peinture dans les récits de voyage de Théophile Gautier $^{1}$. Ce dernier a beaucoup voyagé, dans les musées comme dans le monde. L'intérieur et l'extérieur ne pouvaient que se rencontrer, et parfois se mêler dans son œuvre. Le critique d'art juge la peinture orientaliste des Salons parisiens qu'il parcourt avec le souvenir de ses pérégrinations méditerranéennes. Et, à l'inverse, Gautier voyageur décrit bien souvent l'Orient comme un tableau vivant, comme si la nature finissait par imiter l'art ${ }^{2}$.

Ce lien entre l'art et la réalité semble être consubstantiel à Gautier. Celui-ci en fait état dès son premier récit de voyage (Un Tour en Belgique), en 1836 : il a beau assurer, dès la première page, qu' ' il n'y aura exactement dans [s]a relation que ce [qu'il aura] vu avec [ses]yeux », il avoue quelques lignes plus loin que l'idée de ce voyage lui est venue "au musée ${ }^{3}$. Les déceptions ne se font pas attendre, et Gautier finit par ironiser lui-même sur l'inévitable malentendu consistant à espérer voir se promener dans la rue des figures féminines issues de tableaux de Rubens...

Est-ce à dire pour autant que la peinture soit toujours un filtre, un écran, voir un obstacle à la vision du monde réel, tel que Gautier ambitionne de le décrire, lui qui se définit déjà, en 1843, comme un " daguerréotype littéraire " ${ }^{4}$ ? Rien n'est moins sûr, et l'on pourrait relever de nombreux exemples, dans ses récits de voyage, où son regard de peintre coïncide avec l'intérêt ethnographique du voyageur pour les types humains : la quête du pittoresque (au double sens de ce qui est digne d'être peint et de ce qui est caractéristique, à la fois coloré et singulier), permet, notamment dans Constantinople (1853), de faire se rejoindre ces deux dimensions apparemment contradictoires de la poétique du récit de voyage chez Gautier ${ }^{5}$. Pour ce dernier, la peinture constitue au fond une voie d'accès privilégiée au réel, mais aussi une manière de le reconfigurer imaginairement.

Je voudrais illustrer cette hypothèse en prenant pour corpus un texte peu commenté, celui des feuilletons que Gautier consacra à son séjour en Égypte (1869), et qu'il publia dans le Journal Officiel du 17 janvier au 8 mai 1870. Invité, avec des centaines d'autres personnalités, à l'occasion des fêtes données par le khédive Ismaïl pour l'ouverture du Canal de Suez, il ne fit cependant pas le voyage de la Haute-Égypte auquel participèrent par exemple le peintre Eugène Fromentin, le critique d'art Charles Blanc, ou encore l'écrivain Louise Colet. S'étant cassé le bras

\footnotetext{
${ }^{1}$ Pour un récent état des lieux, voir les articles de Hans Peter Lund («L'art et la réalité dans les Voyages de Gautier ») et d'Alain Guyot («Récréation, devoir et chant du monde. Pour une poétique du voyage et de son récit chez Théophile Gautier») dans le dossier «Panorama Gautier », textes réunis et présentés par Sarga Moussa et Paolo Tortonese, Revue des Sciences Humaines, $\mathrm{n}^{\circ} 277$, janvier-mars 2005.

${ }^{2}$ Sur les liens de Gautier avec la peinture orientaliste, voir Stéphane Guégan, Théophile Gautier. La critique en liberté, Paris, Réunion des musées nationaux, 1997, en particulier le chapitre «Orients : l'insolite de la vie barbare », et Sarga Moussa, « Les Orients de Théophile Gautier : peintres orientalistes et récits de voyage (Espagne, Turquie, Égypte) », dans 48/14. La revue du musée d'Orsay, ${ }^{\circ}$ 5, automne 1997, p. 65-73.

3 Théophile Gautier, Un Tour en Belgique et en Hollande, postface de Stéphane Guégan, Paris, L'école des lettres, 1997, p. 7-8.

${ }^{4}$ Théophile Gautier, Voyage en Espagne, éd. Jean-Claude Berchet, Paris, GF, 1981, p. 197.

${ }^{5}$ Sur ce point, voir notre Relation orientale, Paris, Klincksieck, 1995, chapitre IX.
} 
lors de la traversée de la Méditerranée, Gautier se contenta de voyager, en train, en Basse-Égypte. Et, comme il n'écrivit qu'un très court chapitre sur l'isthme de Suez, c'est essentiellement le trajet entre Alexandrie et Le Caire, ainsi que les pages consacrées à la capitale égyptienne, qu'on examinera ici.

\section{La dialectique de l'art et de la réalité}

Dès l'approche du port d'Alexandrie, Gautier est sensible à la lumière particulière de l'atmosphère orientale :

Çà et là s'épanouissaient des dattiers, comme des plumeaux dont le manche serait planté en terre, et sur tout cela s'étendait un ciel pâle à force d'être lumineux 6 .

Loin du romantisme flamboyant à la Delacroix, loin, également, d'un orientalisme académique comme celui d'Horace $V_{\text {ernet }}{ }^{7}$, ce paysage, sur lequel plane un soupçon d'ironie ${ }^{8}$, renvoie plutôt à l'esthétique d'un Fromentin, le peintre du désert, qui en avait décrit dans ses deux récits "algériens " toutes les nuances et les illusions d'optique, mais qui avait surtout, d'emblée, insisté sur le gris ${ }^{9}$. Cela dit, Gautier utilise toute les nuances les plus subtiles pour décrire les paysages du Delta, dont la terre « étend sa couleur sur toute chose » :

Nous nous disions que, pour la peindre, l'artiste n'aurait besoin sur sa palette que de cette couleur qu'on appelle précisément momie, avec un peu de blanc et de bleu de cobalt pour le ciel. Les animaux eux-mêmes portent cette livrée : le chameau fauve, l'âne gris, le buffle bleu d'ardoise, les pigeons cendrés et les oiseaux roussâtres rentrent dans le ton général ${ }^{10}$.

Le voyageur ne se contente pas d'employer un vocabulaire pictural qui rehausse sa description, mais il construit véritablement celle-ci comme un tableau dont il décompose les différents motifs en leur attribuant une teinte qui contribue à l'harmonie générale. Gautier esthétise le réel, et, symétriquement, il " matérialise » la peinture, en renvoyant à la fabrication du tableau, donc à un processus plutôt qu'à un état fini. Entre l'Égypte vue et l'Égypte peinte, pas de solution de continuité, ce que renforce l'emploi du terme momie, - un mot qui, dans un sens technique, désigne une couleur, mais qui renvoie aussi à la civilisation pharaonique que l'égyptologie du $\mathrm{XIX}^{\mathrm{e}}$ siècle fait émerger, et enfin, de manière indirecte, au Roman de la momie que Gautier avait écrit une dizaine d'années avant d'entreprendre son propre voyage en Égypte.

${ }^{6}$ Théophile Gautier, Voyage en Égypte, éd. Paolo Tortonese, Paris, La Boîte à documents, 1991, p. 35.

${ }^{7}$ Voir Christine Peltre, Les Orientalistes, Paris, Hazan, 1997.

${ }^{8}$ Gautier, comme Flaubert à la même époque, est très conscient des clichés orientalistes (les palmiers !), qu'il s'amuse parfois à démasquer, ce qui n'empêche pas, chez l'auteur du Voyage en Égypte, un amour authentique de l'Orient, - d'où une une tentative de décrire le paysage égyptien en recourant à une esthétique orientaliste relativement nouvelle.

9 Voir par exemple le début d'Un Été dans le Sahara (1857) : "Tout à coup la montagne manque sous vos pieds, l'horizon se dégage, et l'œil embrasse alors à vol d'oiseau, dans toute sa longueur, une vallée beaucoup moins riante, d'un gris fauve qui commence à sentir le feu » (Eugène Fromentin, Euvres complètes, éd. Guy Sagnes, Paris, Gallimard, Bibl. de la Pléiade, 1984, p. 28).

${ }^{10}$ Gautier, Voyage en Égypte, op. cit., p. 48 ; souligné par l'auteur. 
La peinture n'est pas le seul registre artistique mis à conribution pour décrire le Delta. Ainsi, losque Gautier voit le Nil pour la première fois, c'est d'abord à une statue allégorique vue au Louvre qu'il pense. Mais, une fois cette référence mythologique rappelée, c'est à nouveau sous l'aspect d'un tableau en cours d'élaboration que le paysage est représenté ("À peine si le reflet du ciel mettait çà et là sur le luisant de ses vagues tumultueuses quelques légères touches d'azur »), comme pour mieux souligner le caractère mouvant de ce «torrent de limon ${ }^{11}$.

Les villages de fellahs (les paysans égyptiens), aperçus depuis le train, donnent également lieu à une description picturale. Gautier utilise cette fois-ci les ressources rhétoriques de l'ekpharsis en composant un véritable tableau orientaliste qu'il invite son lecteur à produire lui-même. En voici les ingrédients :

Plantez à côté de ces cubes de terre grise un bouquet de dattiers, agenouillez un ou deux chameaux devant ces portes, semblables à des ouvertures de terriers, faites-en sortir une femme drapée de sa longue chemise bleue, tenant un enfant par la main et portant une amphore sur la tête, faites glisser sur tout cela un rayon de soleil, et vous aurez un tableau plein de charme et de caractère, qui ravirait tout le monde sous le pinceau de Marilhat ${ }^{12}$.

On reviendra tout à l'heure sur le peintre orientaliste Marilhat, qui joua, avec Decamps, un rôle déterminant dans le désir d’Égypte de Gautier. Notons pour l'instant la virtuosité avec laquelle ce dernier fait voir tout à la fois le paysage du Delta et une sorte de tableau virtuel, composé sans doute à partir de plusieurs tableaux de Marilhat (par exemple Les Bords du Nil, Benisoef sur le Nil...), si bien que le lecteur contemporain, à travers le souvenir d'expositions qu'il a pu admirer en France, est entraîné tout naturellement, en esprit, sur les traces de Gautier. C'est donc un double objet qui voit le jour avec ce Voyage en Égypte, le texte servant aussi bien à dire le monde perçu par le voyageur qu'à en donner une image médiatisée par la peinture, qui lui confère ainsi une nouvelle réalité.

Gautier ne se contente pas de parsemer son récit de descriptions picturales. Tirant parti de ce nouveau moyen de locomotion que constitue le chemin de fer, il donne à voir un paysage en mouvement, dont les différents "tableaux » s'enchaînent les uns aux autres :

Rien de plus amusant, de plus varié et de plus instructif que cette procession de types qui vont à leurs affaires, et qui s'encadrent tour à tour dans le carreau du wagon comme dans un passe-partout dont on renouvellerait incessamment les gravures ou les aquarelles $^{13}$.

Le cinéma n'est pas encore inventé, mais on y est presque : du Diorama aux frères Lumière, le $\mathrm{XIX}^{\mathrm{e}}$ siècle voit le développement de toute une série de médias destinés à faire voir des images animées, et qui intéressent les écrivains dès l'époque romantique. Gautier tire parti d'une invention datant des années 1830 (le passepartout est à l'origine un cadre à fond amovible pouvant recevoir des gravures de grand format) pour décrire le Delta égyptien en 1869. Mais il ajoute un niveau temporel supplémentaire en comparant un conducteur de chameaux à "Eliézer, serviteur d'Abraham, allant en Mésopotamie chercher une épouse pour Isaac ${ }^{14}$. On oscille donc non seulement entre l'Égypte et la peinture de l'Égypte, mais entre

\footnotetext{
${ }^{11}$ Ibid., p. 53.

${ }^{12}$ Ibid., p. 47-48.

${ }^{13}$ Ibid., p. 49.

${ }^{14}$ Ibid.
} 
l'Orient contemporain et l'Orient biblique, - lequel renvoie lui-même aux peintres orientalistes du XIX siècle : vertige des références, qui affole l'Histoire et conduit le lecteur, par moment, dans un tournoiement spectaculaire...

Gautier, pourtant, n'a pas pour ambition de déconstruire son objet en fictionnalisant le réel. S'il tend, le plus souvent, à donner une représentation esthétisée du monde, les tableaux qu'il évoque entretiennent, de leur côté, un lien étroit avec le motif qu'ils donnent à voir. Si bien que comparer à un tableau de $\mathrm{La}$ Fuite en Égypte l'un des groupes animés qui défilent devant les yeux des passagers ${ }^{15}$, ce n'est pas déréaliser une scène de la vie quotidienne, mais au contraire éclairer celle-ci par l'œuvre des peintres qui, sous prétexte de sujets antiquisants, portent parfois un regard attentif, quasiment ethnographique, sur la population, les costumes, les animaux, etc.

Cette dialectique paradoxale de l'art et de la réalité (la peinture orientaliste servant à authentifier le regard d'un voyageur qui, de son côté, tend à esthétiser le paysage oriental) se trouve mise en œuvre sur un autre plan encore, celui du rapport entre l'Égypte contemporaine et l'Égypte pharaonique. En effet, Gautier, comme nombre de voyageurs du $\mathrm{XIX}^{\mathrm{e}}$ siècle, croit reconnaître dans telle scène de la vie quotidienne une sorte d'agrandissement et d'animation d'un bas-relief qu'il avait d'ailleurs pu consulter bien avant son voyage, par exemple dans les nombreux volumes de planches composant la célèbre Description de l'Égypte (1809-1822) ${ }^{16}$. Quant aux paysans du Delta, Gautier souligne, pour les femmes portant des gargoulettes de Thèbes, leur "galbe de statue », et, pour les hommes accroupis, une posture qui lui rappelle " ces juges de l'Amenthi rangés par file les uns derrière les autres sur les papyrus des rituels funéraires ${ }^{17}$. Seuls les chiens errants, "qui n'avaient pas l'air de se souvenir qu'ils comptaient parmi leurs aïeux l'aboyeur Anubis ${ }^{18}$, échappent à cette image mythifiante d'une Égypte éternelle.

Mais Gautier n'est pas tout à fait dupe de son propre imaginaire. S'il aime à brouiller les frontières entre art et réalité, au point que l'un et l'autre peuvent apparaître comme des composantes d'un même continuum tout à la fois " médiatique " et sensible, il sait aussi varier les points de vue, et même introduire une discontinuité dans le regard qu'il porte sur l'Égypte.

\section{La place de l'Esbekieh}

Avant même d'arriver au Caire, Gautier en a une représentation qui renvoie à un tableau de Marilhat, La place de l'Esbekieh, qu'il avait vu au Salon de 1834, et qui lui avait laissé, écrit-il en 1870, " une sorte de fascination nostalgique ${ }^{19}$. Prosper Marilhat (1811-1847), qui avait voyagé en Orient de 1831 à 1833, avait peint à son retour à Paris un paysage orientaliste sur le mur d'une maison que Gautier habitait,

\footnotetext{
15 «C'était un tableau de la Fuite en Égypte tout fait, il ne manquait aux personnages que le fin cercle d'or au-dessus de la tête. La Vierge, l'Enfant Jésus et saint Joseph devaient avoir ce caractère, et les choses se sont passées ainsi dans la réalité vivante et naïve ; leur équipage n'était pas beaucoup plus riche. Quel dommage qu'un grand peintre, un Pérugin, un Raphaël ou un Albert Dürer ne se soit pas trouvé là » (ibid., p. 51).

16 «Il nous semblait voir marcher ces zones de bas-reliefs, colorés, représentant des scènes d'agriculture, qui parfois décorent les chambres des tombeaux égyptiens » (ibid., p. 56).

${ }^{17}$ Ibid., p. 57 et 58.

${ }^{18}$ Ibid., p. 58.

${ }^{19}$ Ibid., p. 61.
} 
avec quelques autres écrivains et artistes, rue du Doyenné ${ }^{20}$. Nés la même année, le peintre et l'écrivain appartiennent à la même génération romantique. Mais il semble bien que ce soit seulement après-coup que cette première rencontre, d'abord avec le peintre lui-même, puis avec son tableau, ait été présentée comme une sorte de révélation esthétique. Car le jugement de Gautier sur La Place de l'Esbekieh, lors du Salon de 1834, n'avait rien d'enthousiaste: "Son paysage à l'huile nous paraît pécher par la lourdeur et la crudité du ton. Cela peut être vrai ; mais cela n'en a pas l'air: il faudrait aller en Égypte pour être juge compétent. Nous irons. ${ }^{21}$ " II n'empêche que tous les texte ultérieurs où Gautier évoque ce talbeau insistent sur l'aspect fondateur de cette rencontre, celle-ci fût-elle "inventée ». Dans celui de 1848 (la nécrologie consacrée à Marilhat), il va jusqu'à affirmer qu'en voyant ce tableau, il crut reconnaître sa "véritable patrie », - alors même qu'il n'était encore jamais allé en Égypte ${ }^{22}$. Et dans le texte de 1870 (celui des feuilletons parus à la suite des festivités de Suez), il reprend l'exemple de ce même tableau pour illustrer un principe général, celui du souvenir d'une image comme origine d'un profond désir d'altérité:

On se fait, des villes que dès l'enfance on a souhaité voir et que l'on a longtemps habitées en rêve, un plan fantastique bien difficile à effacer, même quand on se trouve en face de la réalité ; la vue d'une gravure, d'un tableau en est souvent le point de départ. ${ }^{23}$

D'ailleurs, tout en s'efforçant de décrire aussi précisément que possible la place de l'Esbekieh, Gautier ne renonce pas pour autant à comparer ce qu'il voit en 1869 avec le souvenir ébloui du tableau de Marilhat. C'est significativement la description de celui-ci qui vient en premier dans le récit de voyage :

Ce tableau, qui éteignait autour de lui les toiles représentant une nature plus sobre, était d'une incroyable férocité de couleur. Sur un ciel d'un bleu cru, dont l'outremer tournait à l'indigo, se découpaient deux arbres immenses de l'espèce mimosa Nilotica, avec un pied monstrueux qu'on aurait cru fait d'une botte de colonnes tordues, et des branches qui étaient elles-mêmes d'énormes troncs formant des coudes bizarres et portant des masses de feuillage à couvrir une forêt. Ces deux arbres occupaient à eux seuls presque tout le cadre et, sous l'ombre qu'ils projetaient, on entrevoyait dans l'obscurité bleuâtre une "sakkieh » manœuvrée par des buffles, une femme ayant une cruche d'eau sur la tête, divers personnages accroupis, et un Arabe juché sur un chameau. Plus loin, vers la gauche, s'épaulaient les unes contre les autres, dans tout le laisser-aller oriental, les maisons arabes qui bordent la place, avec leurs moucharabiehs, leurs étages en surplomb, leurs encorbellements soutenus de poutrelles, et tous les détails caractéristiques que n'avait pas encore émondés le progrès, ami des lignes droites et des surfaces planes. Un palmier levait au-dessus des maisons son plumeau de feuilles, et derrière les arbres, sous la voûte de leur feuillage, on entrevoyait une autre rangée de

${ }^{20}$ Gautier évoque cet épisode dans la notice nécrologique qu'il consacra à Marilhat (Revue des Deux Mondes, $1^{\text {er }}$ juillet 1848), reproduite dans le Voyage en Égypte, op. cit., p. 103. Il a souvent commenté des tableaux de Marilhat, y compris après la mort de ce dernier : voir Exposition de 1859, éd. Wolfgang Drost et Ulrike Henninges, Heidelberg, Winter, 1992, p. 39 et passim. Voir par ailleurs l'article de Frans Amelinckx, «Théophile Gautier et Marilhat : peintures, textes et contextes », dans Bulletin de la Société Théophile Gautier, 1982, t. I, p. 19.

${ }^{21}$ La France industrielle, $\mathrm{n}^{\circ}$ 1, avril 1834, cité par F. Amelinckx, "Théophile Gautier et Marilhat... », BSTG, op. cit., p. 3.

${ }^{22}$ Gautier, «Marilhat », dans Voyage en Égypte, op. cit., p. 104.

${ }^{23}$ Gautier, Voyage en Égypte, op. cit., p. 61. 
bâtiments formant le fond de la place et surmonté d'un minaret ${ }^{24}$.

Les deux grands arbres occupent effectivement une très grande partie du tableau ${ }^{25}$, ce qui confère un rôle subordonné à tous les éléments renvoyant habituellement à l'exotisme orientaliste (palmier, mosquée, chameau, femme avec une cruche...), placés soit dans le lointain, soit au premier plan, mais occupant une place très réduite. Autre élément surprenant: l'impression d'ensemble est celle d'une ville encore très proche de la nature, et qui ne correspond guère à l'image d'une capitale déjà populeuse, telle qu'on peut se la représenter grâce au récit des voyageurs en Égypte contemporains de Marilhat ${ }^{26}$. Celui-ci donne donc, vraisemblablement, une représentation très personnelle de la capitale égyptienne, qui vaut comme métonymie d'un Orient " immobile ». Or c'est cela même qui plaît à Gautier, lequel retrouve dans ce tableau « tous les détails caractéristiques [des maisons arabes] qui n'avaient pas encore été émondés par le progrès ". Autrement dit, lorsqu'il évoque La Place de l'Esbekieh, il a déjà devant lui... la place de l'Esbekieh, celle qui va apparaître dans un second temps, dans le récit, et dont la description témoignera des changements urbains intervenus en quelques dizaines d'années :

On en a fait un grand square à l'européenne, divisé par de larges voies en compartiments réguliers, bordées de légères palissades de roseaux ou de nervures de palmiers, qu'on espère vendre pour y bâtir des maisons à peu près comme le parc Monceau, tout en réservant une partie du terrain pour la promenade. [...].

Des arbres énormes, - mimosas et sycomores, - parmi lesquels nous reconnûmes sans peine ceux qui avaient posé pour Marilhat, agrandis encore par le temps écoulé, garnissaient le milieu de la place avec leurs dômes de feuillage, d'un vert si intense qu'il paraît presque noir. Sur la gauche s'élevait, comme dans le tableau, une rangée de maisons où l'on distinguait, parmi quelques bâtisses neuves, d'anciennes habitations arabes plus ou moins modernisées: un grand nombre de moucharabiehs avaient disparu ; il en restait cependant assez pour conserver à ce côté de la place le caractère oriental. Nous devons avouer que sur une des premières maisons de la file, peinte de ce bleu qu'en France on appelle bleu perruquier, se lisaient en grandes lettres ces mots : Maison de l'ancienne cave populaire ${ }^{27}$.

Ce qui frappe dans cette description, c'est le caractère hybride de l'espace, dont les signes occidentaux et orientaux alternent sans pour autant se mélanger. II s'agit donc moins, pour Gautier, d'un " métissage " symbolique, que d'une juxtaposition problématique d'éléments hétérogènes. La place de l'Esbekieh est au fond perçue simultanément de deux façons, c'est-à-dire à travers le filtre esthétisant du tableau de Marilhat, et, de manière réaliste, comme une manifestation des transformations urbaines de la capitale égyptienne. Cette "double vue », fondée tant sur le souvenir que sur le regard, permet de donner une profondeur temporelle à la description. En effet, si l'écrivain cherche à repérer, dans Le Caire tel qu'il le voit, en 1869, des

\footnotetext{
${ }^{24}$ Ibid., p. 61-62.

${ }^{25}$ Le tableau de Marilhat, qui date de 1833, s'intitule précisément «Vue de la place de l'Esbekieh et du Quartier Copte, au Caire ». Il s'agit d'une huile de 107 X $161 \mathrm{~cm}$., qui a été vendue à Londres, chez Sotheby's, le 12 octobre 2000. Ce tableau, qui avait disparu de la circulation depuis longtemps, peut être vu sur le site Artnet.

26 Voir par exemple les Lettres sur l'Orient (1829) de Marie-Théodore Renoüard de Bussierre : «La foule se porte en masse dans les rues passantes : on se heurte, on s'écrase ; personne ne songe à s'éviter... » (dans Sarga Moussa, Le Voyage en Égypte, Paris, Laffont, coll. « Bouquins », 2004, p. 86).

${ }^{27}$ Gautier, Voyage en Égypte, op. cit., p. 63-64.
} 
indices d'une permanence renvoyant à la représentation qu'en avait donnée le peintre en 1833, il ne cache pas non plus l'européanisation d'une ville qui, certains voyageurs des années 1870-1880 en témoignent, fait l'objet d'une " haussmannisation » s'inspirant de celle que connaît Paris sous le Second Empire ${ }^{28}$. Bien entendu, Gautier n'attribue pas la même valeur à ces deux visions du Caire : s'il ironise sur une enseigne française incongrue dans ce décor, la «fascination nostalgique » pour la capitale égyptienne, qu'il disait avoir éprouvée en regardant le tableau de Marilhat, se reporte, quelques décennies plus tard, sur le tableau luimême, dont la vue de la place de l'Esbekieh réactive le souvenir. Ce n'est du reste pas seulement l'art qui a ici un statut supérieur, mais, plus précisément, l'art en tant qu'il renvoie à un Orient encore intouché par la civilisation occidentale. Comme il l'avait fait en 1853 dans Constantinople ${ }^{29}$, Gautier sélectionne tout ce qui, dans le paysage d'une ville orientale, apparaît comme la survivance d'une beauté native, antérieure à une modernisation dont il fuit précisément la laideur envahissante.

\section{Orientaliser l'Orient moderne}

Dans son célèbre essai sur L'Orientalisme, Edward Saïd reproche à la plus grande partie des écrivains anglais et français du XIX ${ }^{e}$ siècle, et en particulier aux voyageurs (mais il ne prend quasiment jamais en considération l'œuvre de Gautier), de donner de l'Orient une image déformante, fondée sur des préjugés et des stéréotypes le plus souvent dépréciatifs (Arabes "cruels", musulmans "paresseux ", etc.). Cette manière d'« orientaliser l'Oriental " ${ }^{30}$ est à coup sûr critiquable, et Saïd a eu raison de mettre en évidence, pour en démasquer les implications idéologiques, un imaginaire occidental témoignant d'une forme d'ethnocentrisme, donc de méconnaissance de l'autre. Pour autant, il faut se garder de condamner en bloc toute représentation " orientaliste »: si Gautier donne de l'Égypte une vision fortement médiatisée (en l'occurrence par un tableau et par le souvenir qu'il en a), cela ne signifie nullement qu'il cautionnerait un discours " civilisateur » en accord avec l'impérialisme des grandes puissances européennes rivalisant pour dominer la Méditerranée, - tout au contraire: sa façon à lui d'« orientaliser l'Orient » a pour fonction de relégitimer celui-ci.

Comme Loti le fera après lui, notamment dans La Mort de Philæ (1908), Gautier stigmatise tout ce qui, en Égypte, lui paraît relever d'une dénaturation du paysage oriental due à la présence européenne. Mais ce faisant, il fait voir deux réalités qui coexistent tant bien que mal dans un même espace. À preuve la façon dont il décrit Tantah, ville du Delta où s'arrête le train qui emmène les invités du khédive :

Aux maisons de style arabe, avec leurs moucharabiehs et leurs manches d'air en forme d'auvent, se mélangent ces constructions de style italien orientalisé qu'affectionnent les partisans du progrès et des idées nouvelles, mais qui font le désespoir de l'artiste, étalant leurs façades badigeonnées de couleurs tendres, ocre, saumon ou bleu de ciel...

Gautier ne voit pas (et ne veut pas voir) que la présence italienne en Égypte est ancienne, et qu'elle fait depuis bien longtemps partie d'un Empire ottoman composé

\footnotetext{
${ }^{28}$ Sur cette haussmannisation voulue par les khédives, voir, dans notre anthologie Le Voyage en Égypte, les textes d'Adolf Ebeling et de Blanche Lee Childe (op. cit., p. 913 et suiv.).

${ }^{29}$ Voir Théophile Gautier, Constantinople et autres textes sur la Turquie, éd. Sarga Moussa, Paris, La Boîte à documents, 1990.

${ }^{30}$ Edward Saïd, L'Orientalisme. L'Orient créé par l'Occident, trad. fr., Paris, Seuil, 1980, p. 66 et suiv.
} 
de multiples communautés, dont certaines sont issues d'Europe. Parce qu'il a en tête le tableau de Marilhat qui, pour lui, inscrit l'Égypte dans une sorte de " naturalité » primitive, mais aussi parce qu'il répond aux besoins d'un public français avide d'exotisme ${ }^{31}$, Gautier recherche systématiquement tout ce qui lui paraît relever d'un Orient « authentique ». Deux stratégies de représentation sont alors possibles. Soit il oppose une Égypte « orientale » à une Égypte « européanisée » pour valoriser la première, comme il le fait dans la description de Tantah, soit il refoule tout ce qui lui semble relever d'une hybridité esthétique dommageable, au profit d'une orientalité conforme à ses propres attentes, comme à la fin de la description qu'il fait de la place de l'Esbekieh: "Les arbres du square nous masquaient les constructions nouvelles, les théâtres du Cirque, de l'Opéra-Italien et de la Comédie-Française, et de cette façon notre rêve n'était pas trop dérangé ${ }^{32}$. " Mais on voit le caractère profondément ambigu de ce refoulement, qui énumère soigneusement tous les édifices masqués, ce qui, du coup, les fait exister dans la conscience du lecteur, puisqu'ils appartiennent à son univers de référence. Gautier veut tout à la fois poursuivre son rêve et faire comprendre qu'il n'en est pas dupe ${ }^{33}$. Comme Nerval, dont il est proche par bien des aspects, il se plaît à poursuivre un fantôme tout en jetant parfois le soupçon sur sa propre démarche, voire en ironisant sur sa quête de "pureté » orientale sans cesse démentie par un réel en perpétuelle évolution, donc intrinsèquement hybride.

Gautier n'est pourtant pas insensible à la diversité propre d'un Empire ottoman composé d'une mosaïque de communautés ayant chacune sa langue, sa culture et sa religion. Mais il tend à mettre en évidence les éléments qui, d'un point de vue ethnographique, caractérisent ce monde dans sa différence par rapport à l'Europe. Voici comme il décrit le spectacle de la rue, qu'il observe avec une lunette depuis la véranda de l'hôtel Sheppeard :

II y avait là des drogmans [guides-interprètes], la plupart grecs ou cophtes, coiffés du fez, en petite veste soutachée et en larges pantalons ; des cawas [gardes attachés à un consulat], richement costumés à l'orientale, le sabre courbe sur la cuisse et le kandjar [poignard à longue lame] à la ceinture, tenant à la main une canne à pommeau d'argent ; des domestiques indigènes en turban blanc et en robe blanc ou rose ; des petits nègres, les jambes et les bras nus, vêtus de courtes tuniques rayées de couleurs vives; des marchands offrant des couffiehs [turbans], des gandouras [tuniques sans manches] et des étoffes d'Orient fabriquées à Lyon ; des photographes faisant voir des vues d'Égypte et du Caire, ou des reproductions de types nationaux ; sans compter les voyageurs euxmêmes qui, venus de toutes les parties du monde, méritaient bien un peu d'attention ${ }^{34}$.

Si l'on reprend les différents éléments de cette description, on s'aperçoit qu'on

${ }^{31}$ Sur cette question, voir les travaux de Jean-Marc Moura, notamment La Littérature des lointains : histoire de l'exotisme européen au XX siècle, Paris, Champion, 1998.

${ }^{32}$ Gautier, Voyage en Égypte, op. cit., p. 64. Sur ce paradoxe d'un regard qui pose un voile sur le réel, voir Alain Buisine, L'Orient voilé, Cardeilhan, Zulma, 1993 (il n'y est cependant pas question de Gautier).

${ }^{33}$ Nous rejoignons ici ce qu'écrit Paolo Tortonese dans son introduction aux CEuvres de Théophile Gautier, à propos de ses textes de fiction : «En lisant Gautier, nous ne pouvons oublier qu'il est en train de jouer avec nous le jeu de la littérature. Jamais il ne nous permet de nous faire illustion au point d'oublier que nous sommes en train de lire un roman, non de vivre un rêve. Mais en même temps, il entretient chez nous la conscience de la raison pour laquelle on lit des romans : parce qu'on voudrait vivre dans les rêves » (Paris, Laffont, «Bouquins », 1995, p. II-III).

${ }^{34}$ Gautier, Voyage en Égypte, op. cit., p. 65. 
part des chrétiens orientaux pour aboutir aux voyageurs européens, mais que l'objet principal est constitué par des groupes humains renvoyant à l'Orient musulman (les cawas et les domestiques) ; les Noirs, quant à eux, rappellent que l'Égypte est aussi un pays africain, et que la plus grande partie de son territoire est tournée vers le Sud. D'autre part, Gautier superpose à ce tableau ethnographique une description vestimentaire qui en exotise les différents éléments : les Grecs et les Coptes portent certes le costume de la réforme ${ }^{35}$, mais celui-ci apparaît surtout comme le signe d'une réjouissante exubérance de couleurs qui tranche avec la mode du complet noir dans la France bourgeoise du XIX ${ }^{\mathrm{e}}$ siècle. Enfin, les photographies ne semblent pas affectées d'un coefficient négatif, dans la mesure où elles mettent en valeur l'Égypte, fût-ce à travers des technologies nouvelles venues d'Europe. Quant aux voyageurs, censés mériter "un peu d'attention », on observera qu'ils ne sont même pas décrits...

La peinture orientaliste, qui a hanté Gautier toute sa vie, s'avère bien autre chose, dans son Voyage en Égypte, qu'un élément relevant de la quête du pittoresque, - une dimension qu'il a pourtant lui-même beaucoup exploitée dans ses récits de voyage antérieurs, notamment dans Constantinople. En 1869, la référence picturale constitue en quelque sorte un instrument de reconfiguration du réel, qui permet au voyageur de sélectionner ce qui, dans un paysage urbain en pleine mutation, renvoie l'image d'un Orient non encore «pollué » par la présence européenne : le tableau de Marilhat, qui présente l'avantage de dater d'une période bien antérieure à celle du voyage de Gautier en Égypte, a ainsi pour fonction de délégitimer la présence européenne en Orient, - celle-là même qui motive l'œuvre de Lesseps, à laquelle les fêtes données pour l'ouverture du canal de Suez rendent hommage. Ces feuilletons ont par conséquent une dimension critique d'autant plus remarquable que Gautier se trouve parmi les invités du khédive, et qu'il ne cède pas au discours euphorique sur la célébration du " mariage de l'Orient et de l'Occident ". Le double regard que le voyageur porte sur l'Égypte est à l'opposé d'une " voyance » tournée vers l'avenir et le progrès : il conduit à réévaluer l'Orient, non en fonction de sa capacité à s'adapter à la modernité occidentale, mais au contraire à lui résister pour offrir au monde une beauté première.

Sarga MOUSSA (CNRS, UMR LIRE)

\footnotetext{
${ }^{35}$ Le fez remplace le turban à Constantinople à partir des années 1830. En Égypte, on parle plutôt du tarbouche, qui fait partie de ce costume censé refléter l'ouverture des vice-rois, puis des khédives, à la modernité européenne.
} 